\title{
EFFECT OF CAD/CAM VERSUS CONVENTIONAL ANTERIOR REPOSITIONING SPLINTS ON THE MANAGEMENT OF TEMPOROMANDIBULAR JOINT DISC DISPLACEMENT WITH REDUCTION: A RANDOMIZED CONTROLLED CLINICAL TRIAL
}

\author{
Iman A. El-Asfahani* and Sahar A. Kortam**
}

\begin{abstract}
Purpose: The aim of the current study was to investigate the effect of CAD/CAM versus conventional anterior repositioning splints (ARS) on the management of temporomandibular joint disc displacement with reduction.
\end{abstract}

Materials and methods: Twenty patients ( 15 females and 5 males) diagnosed (clinically and by magnetic resonance imaging (MRI)) with disc displacement with reduction (DDWR) were selected from the outpatient clinic of oral and maxillofacial surgery department, Faculty of Dentistry, Beni-Suef University. Patients were randomly divided into two groups; Group I patients received ARS constructed following the conventional technique. Group I patients had 8 normal (control) and 12 DDwR joints as scanned by MRI. Group II patients received ARS constructed by CAD CAM technique. Group II patients had 6 normal(control) and 14 DDwR joints as scanned by MRI. Subjective symptoms were evaluated using temporomandibular disorder (TMD) questionnaire before treatment (baseline), 1-, 3- and 6-months post- insertion. Moreover, MRI measurements of the disc-condyle angle and the disc/condyle positions were performed for normal (control) and DDwR joints in group I and group II. There measurements were done before treatment, immediately at the time of splint insertion and finally after 6 months post-insertion.

Results There was no statistically significant difference between the scores of TMD questionnaire items between group I and group II at baseline and different follow up visits. Likewise, there was no statistically significant difference in disc-condyle angle and disc/condyle positions (MRI measurements) between DDwR joints in group I (conventional ARS) and group II (CAD/CAM ARS) at pretreatment, immediately at the time of splint insertion and after 6 months post-insertion.

Conclusion Within the limitations of this study, there was no significant difference between the effect of CAD/CAM versus conventional ARS on the management of DDwR. Hence, the CAD/CAM ARS can provide a good substitute to the conventional ARS in treatment of DDwR eliminating human errors and saving time. Moreover, the superior effect of ARS in the management of DDwR could be related mainly to the design of the splint rather than the method involved in the construction of the splint.

* Lecturer of Removable Prosthodontics, Faculty of Dentistry, Minia University

* Lecturer of Removable Prosthodontics, Faculty of Dentistry, Beni-Seuf University 


\section{INTRODUCTION}

Temporomandibular disorders (TMDs) present as a group of pathological conditions regarding derangements of the condyle-disc complex and having their origin in the musculoskeletal structure of masticatory system. The symptoms and signs of TMD can be joint sounds, headaches, pain, restricted or abnormal jaw movements and/or locking of the jaws ${ }^{1}$. There are four basic categories of articular disc displacements according to the Research Diagnostic Criteria for Temporomandibular Disorders (RDC/TMD); disc displacement without reduction with limited opening, disc displacement without reduction without limited Opening, disc displacement with reduction, disc displacement with reduction with intermittent locking ${ }^{2}$.

It is confirmed that the disc displacement with reduction (DDwR) is the most widespread derangement of the condyle-disc complex ${ }^{3}$. The DDwR is defined by the RDC/TMD as an intracapsular biomechanical disorder when - in closed mouth position- the disc takes an anterior position in relation to the condylar head, then the disc reduces with opening of the mouth. Masticatory muscle contracture can cause DDwR. Snapping, clicking, noises or popping might exist with disc reduction. These audible signs are indication of disc displacement with reduction. ${ }^{2}$ Several studies reported that pain is a concurrent symptom of displacement of the temporomandibular joint (TMJ) disc ${ }^{4,5}$. Once the disc is displaced anteriorly, the rear disc ligaments are overextended, damaged, and the bilaminar zone is compressed; pain could be created. The pain is positioned in the area of the TMJ and escalates once the patient opens his mouth and masticates food ${ }^{4}$.

Correction and treatment of abnormal disccondyle relationship ought to be performed in symptomatic patients to avoid serious complications to the TMJ. Since disc dislocation will not be corrected naturally and early recapture (returning to normal position) of the reducing disc must be done before it is irreversibly malformed. Surgical or conservative approaches are used in treatment of TMD. The conservative approach is often preferred by most of patients in the form of intraoral occlusal appliances to decrease pain and improve function. Different types of intraoral splint designs have been used (e.g. stabilization, anterior bite, palatal and anterior repositioning splints). The effectiveness of occlusal splints in reducing symptoms was reported to be between $70 \%$ to $90 \%{ }^{6}$.

Several studies proved the effectiveness of anterior repositioning splint (ARS) in treatment of TMJ discs displacements ${ }^{7-10}$. The ARS is a removable, convenient, and simple device maintaining the patient's bite in a protruded edge to edge relation. The ARS aid in directing the condyle anteriorly in the glenoid fossa (i.e., protrusive position) temporarily during treatment to assist the adaption of the retrodiscal tissues ${ }^{11}$. Additionally, ARS can also reduce mechanical stresses in the TMJ rising from instant physiologic improvement in the disc-condyle relation $^{12}$ therefore, enabling regenerative remodeling of $\mathrm{TMJ}^{13}$. The improved condyle-disc relationship with ARS was thought to be achieved primarily by the anteroinferior movement of the condyle ${ }^{14}$. The chief treatment objective is to eradicate joint sounds by recapturing the disc; then a harmonized, painfree and smooth motion range is often attained ${ }^{15}$.

The advent of digitalization enables the construction of oral appliances and prostheses by the computer-aided design/computer-assisted manufacture (CAD/CAM) with subtractive and additive methods ${ }^{16}$. The two methods of manufacturing can be either grinding and milling in subtractive manufacturing or selective laser sintering, stereolithography, photo-curing print, fused deposition modelling in additive (3D printing) manufacturing ${ }^{17}$. The occlusal splints can be either fabricated conventionally or by means of a complete digital workflow using additive as well subtractive $\mathrm{CAD} /$ CAM techniques ${ }^{18}$. Conventional methods of ARS 
construction are very technique sensitive often leading to poor fit and need prolonged chairside time to be adjusted and attain passive fit with a proper occlusal scheme ${ }^{19}$. Furthermore, these splints can be more susceptible to fracture and/or failure throughout usage which causes discomfort for both patient and dentist $\mathrm{t}^{20}$. In contrast to conventional procedures with different human errors, the use of CAD/CAM in splint construction eradicate those errors (inherent in the casting and other technical processes), providing high material quality, less construction time and the option of manufacturing duplicate splints ${ }^{18}$. CAD/CAM splints have high level of predictability at the clinical and technical levels with the use of nontraditional materials which are not feasible for casting. CAD/CAM splints have been appealed to superior to conventional due to outstanding material and construction methods ${ }^{19,21}$. In a systematic review and meta-analysis, it was concluded - despite the limited number of included studies- that CAD/ CAM splints might be a good substitute construction modality for TMDs patients capable of providing the cost of this treatment option ${ }^{22}$.Unfortunately, the quality of TMD randomized controlled clinical trials is modest and rare, and that better-quality and well-designed researches in this issue are required ${ }^{23}$. The studies evaluating CAD/CAM technique in construction of splints for treatment of TMDs are rare in the literature ${ }^{19,21}$. Therefore, comparing the efficacy of conventional versus CAD/CAM ARS can be beneficial due to the advantages offered by CAD/CAM technique. Moreover, the current study is one of the few randomized controlled clinical trials comparing $\mathrm{CAD} / \mathrm{CAM}$ versus conventional ARS in the management of disc displacement with reduction (DDWR) cases in TMDs.

The effect of ARS on TMJ disc position was investigated in few studies based on clinical experience $^{7,9}$. Nevertheless, limited number of studies used imaging modalities to determine the actual disc recapture ${ }^{10,14,25}$ It is more accurate and precise to confirm ARS disc recapture before splint therapy and after completion of treatment. Also, the association and correlation of MRI with subjective symptoms evaluation can provide an effective method of assessment of ARS. Thus, the present study compared the effect of CAD/CAM versus conventional ARS in treatment of TMDs radiographically using magnetic resonance imaging (MRI) and a TMD questionnaire.

\section{MATERIALS AND METHODS}

\section{Selection of Patients:}

Twenty patients (15 females and 5 males) complaining of TMD were selected from the outpatient clinic of oral and maxillofacial surgery department, Faculty of Dentistry, Beni-Suef University. The range of their ages was from 20 up to 40 years. The effect of confusing variables including marked skeletal/ occlusal discrepancies and systematic diseases was reduced by following certain exclusion criteria. Excluded subjects were: (a) older than 40 years; (b) pregnant; (c) with congenital abnormalities or dentofacial deformities; (d) suffering from recent oro-facial or cervical trauma; (e) with major psychological disorders; (f) with complete or partial dentures that may affect occlusal splint support; ( $\mathrm{g}$ ) received previous TMD treatment or taken analgesics, muscle relaxant or anti-inflammatory drugs which may influence the results; (h) Presence of crossbite, open bite, deep overbite or large overjet; (i) rheumatic or degenerative joint diseases and (j) periodontal diseases or bad oral hygiene. However, patients enrolled were consistent through strict inclusion criteria that were based on Research Diagnostic Criteria for TMD (RDC/TMD); Axis I (clinical aspects of TMD) Group II (DDWR that has been diagnosed clinically and by MRI for the right and left joints). Subjective symptoms of RDC/TMD (Axis II) must be evaluated using TMD questionnaire. The patients contributed in the study were informed about the nature of the study and signed an informed 
patient consent. The study was accepted by the ethical committee of the Faculty of Dentistry, BeniSeuf University

\section{Patients diagnosis}

a) Clinical diagnosis: was done using the following schedule: (1) palpation of TMJ area on both sides for each patient detecting TMJ tenderness. Muscles of mastication as well as sternomastoid and trapezius muscles were assessed to exclude myofascial pain syndrome. (2) Audiation of clicking, popping and/or crepitus during mouth opening and reciprocal closing (3) Limited mouth opening might be present in some cases and it was evaluated by measuring the distance between upper and lower incisors in mm (4) Mandibular protrusive test was done by placing wooden tongue depressor (1.5 mm thickness) on the upper anterior teeth, and then the patient was directed to protrude the mandible till edge to edge position where the lower incisors touched the tongue depressor . The patient was stopped at the previous position and opened his mouth again. The patients whose joint sounds disappeared by means of the previously mentioned maneuver were good candidates for ARS therapy.

b) TMD Questionnaire: Subjective symptoms (RDC/TMD -Axis II) were evaluated using a TMD questionnaire ${ }^{26}$. It consists of five questions investigating the features of TMJ pain, masticatory muscle pain, TMJ sounds (clicking/popping or crepitus), difficulty during mouth opening (MO) and oral parafunctional habits (bruxism or clenching) as shown in table (1). The questionnaire was translated into Arabic language to be understood by patients. The severity of each symptom mentioned in that questionnaire was evaluated by the patient using Visual Analogue Scale (VAS). The individuals independently rated the current intensity of symptoms on a $100-\mathrm{mm}$ line by marking a point on the line between the two extremes where the left extreme of the scale is marked "nothing felt" and the right one is marked "the worst imaginable sign". The distance from the start line to the marked point was measured with a ruler to determine the complaint intensity. The total score for each response in questionnaire was calculated, higher scores indicated increased severity of symptoms and complaints ${ }^{27}$.

TABLE (1) The items of TMD questionnaire

Have you ever observed sounds during closing or opening your jaw? i.e. Crepitus, Clicking or popping

Have you ever had pain in the joint around your ears?

Have you ever had pain around your temple, cheeks, or jaw?

Have you ever experienced any difficulties during opening your mouth to the point where two fingers can fit inside?

Do you suffer from any oral parafunctional habits? i.e. Clenching or Bruxism

c) Magnetic Resonance Imaging (MRI): To confirm the diagnosis of DDwR in the patients, MRI was performed for each patient for right and left temporomandibular joints, hence determining the exact number of normal and DDwR (temporomandibular joints having disc displacement with reduction) joints based mainly on determining the exact angle between the disc and condyle mainly as well as the measurements of disc and condyle spatial positions. MRI scan with a 1.5-Tesla MR scanner (Phantom Healthcare Pvt. Ltd. Sector 27c Faridabad, India) with TMJ surface loops was done. The patients were kept in a supine position with their heads situated with the Frankfurt plane perpendicular to sagittal plane. The central beam was in line with the sagittal plane. An initial low-resolution T1-weighted (TR300 ms; TE $10 \mathrm{~ms}$ ) axial localizing scan was 
followed by Proton-weighted (TR $1760 \mathrm{~ms}$, TE $15 \mathrm{~ms}$ ) oblique sagittal scan acquired vertical to the long axis of each condyle. The field of view was $12 \times 12 \mathrm{~cm}$, and matrix size was $512 \times 512$. Slice thickness and interslice spacing were set at $2 \mathrm{~mm}$ and $1 \mathrm{~mm}$ respectively. Disc and condyle angles/positions were evaluated using two central and sagittal MRI images of the TMJs by a single observer.

I. Disc-condyle angles measurement: was done using methodology designated by Drace and Enzmann ${ }^{28}$. Figure (1a) showed that Point $\mathrm{H}$ is the centric point of condyle head along its horizontal axis, and point $\mathrm{P}$ is at the middle of the posterior margin of the posterior band of the disc. Two lines were drawn, where the line 1 was passing through point $\mathrm{H}$ perpendicular to the Frankfort horizontal plane and the line 2 was extended between point $\mathrm{H}$ and point $\mathrm{P}$. The angle between Line 1 and Line 2 is the disc-condyle angle whose normal range is between $-15^{\circ}$ and $+15^{\circ},{ }^{32}$ and any disc-condyle $>+15^{\circ}$ means the presence of anterior disc displacement. ${ }^{29}$ Images from the MRI scanner were obtained, and GIMP software (GNOME Organization, Massachusetts, USA) was used for computing and quantifying the disc-condyle angles.

\section{The disc and condyle spatial positions: $\mathrm{X}-\mathrm{Y}$} axes (coordinates) were used to determine positions of disc and condyle. Line 3 was made connecting between the tangent from lowest part of the articular tubercle (point $\mathrm{L}$ ) to the highest edge of the porus acusticus externus (point E). $\mathrm{X}$-axis was drawn passing through the highest point of the glenoid fossa (point F) parallel to the line 3. Y-axis was drawn from the point $F$ perpendicular to the $\mathrm{X}$-axis and the point $\mathrm{F}$ was considered as the origin of coordinates. The condyle and disc positions were points $\mathrm{H}$ and $\mathrm{P}$ respectively, and their $\mathrm{X}$ and $\mathrm{Y}$ coordinates were measured in millimeters using GIMP software based on the measurement scale as shown in figure (1b).
All temporomandibular joints of patients were scanned during the first MRI exposure (pretreatment) in maximum intercuspation and maximum opening respectively to verify disc position (anterior to the condyle) in maximum intercuspation and its reduction in maximum opening. The normal and DDwR joints were assessed and registered. Likewise, Disc-condyle angles and positions were evaluated in this stage. The MRI data were assessed by a trained observer blinded to the clinical data. The intra-class correlation coefficient (ICC) was used to determine the intra observer reliability. The mean intra-observer ICC of $>0.93$ was confirmed for the entire variables.

\section{Patients Grouping}

Patients were randomly divided into two equal groups using a statistical software program (Minitab 17.0, Pennsylvania, USA). The randomization procedure was performed blindly. The allocation ratio was $1: 1$ for the conventional and $\mathrm{CAD} / \mathrm{CAM}$ ARS splint groups the results of the randomization were recorded and preserved for each patient for the following patient grouping. According to the type of splint received, the patients were divided into two groups (ten patients each); Group I: patients received conventional ARS and Group II: patients received CAD/CAM ARS.

\section{Splints construction (fabrication)}

a) Group I (Conventional ARS): Maxillary and mandibular impressions with reversible hydrocolloid (Cavex, fast set, Holland) were made for each patient. Impressions were poured with extra hard stone (Kimberlite, Type IV Dental stone -Spain) to obtain casts. The maxillary cast was mounted on a semi-adjustable articulator (Bio-art semi-adjustable articulator. A7 Plus. Brazil) by means of maxillary face bow (Bio-art face bow, Brazil). Interocclusal bite registration of maximum intercuspation using $3 \mathrm{~mm}$ of modelling wax (Cavex, set up soft Modelling wax, Holland) was done to mount the lower cast 
figure (2a). Protrusive interocclusal record with modelling wax was performed and transferred to the articulator to adjust horizontal condylar guidance. On the upper cast, the limits of splint were drawn such that the splint should cover $2 \mathrm{~mm}$ of the labial and buccal surfaces of all maxillary teeth and provide full coverage to the palatal surface of anterior and posterior teeth. Maxillary cast was then placed on surveyor to make wax block out of all undercuts beyond the drawn borders of splint. The surveyed maxillary cast was duplicated and then remounted on the articulator and then two sheets of pink wax were adapted on it to make the waxing up of ARS following the previously mentioned limits. Once the waxing up was completed, the duplicated maxillary cast was removed from the articulator to be invested in a flask where the waxed-up splint was processed using clear hard heat cured acrylic resin (Acrostone, Egypt). After Finishing of acrylic splint, it was refitted on the mounted working maxillary cast to make occlusal adjustments. The splint was finally seated in the patient mouth to check seating, stability and retention. The occlusion was verified using the articulating paper. During protrusion using ARS, the posterior teeth should be dis-occluded by the even thickness of the splint $(4 \mathrm{~mm})$ and only incisal edges of upper and lower anterior teeth were allowed to make contact through a narrow trough made anteriorly in the splint. The patient was instructed to wear the splint 8 hours per day for 6 months figure (2b).

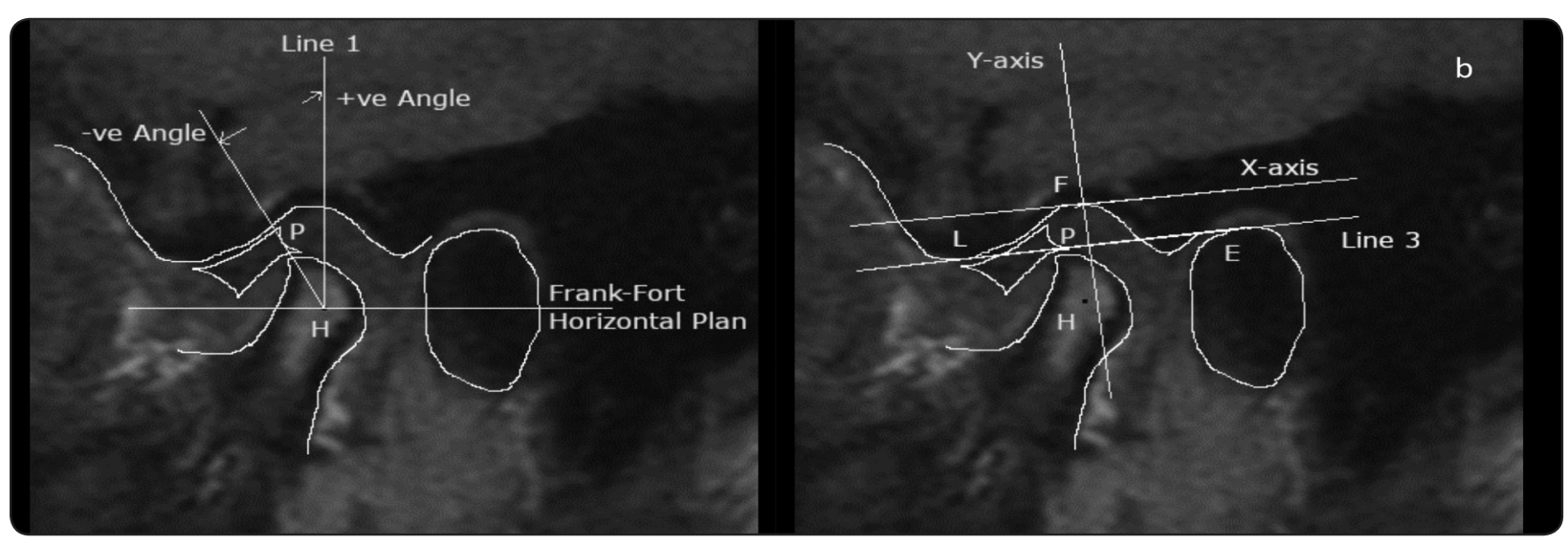

Fig.(1) a) measurement of disc-condyle angle, b) Coordinate measurements for disc and condyle positions.

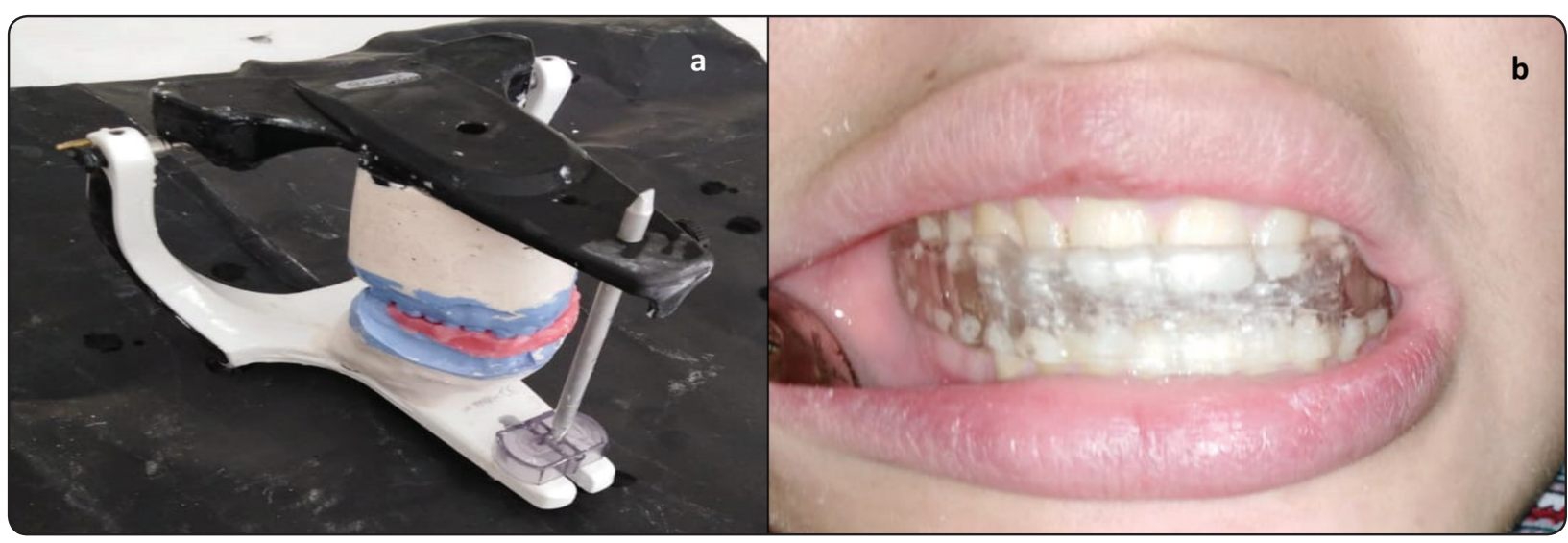

Fig. (2) a) casts mounted on the articulator, b) Conventional ARS intraorally 
b) Group II (CAD/CAM ARS): The upper and lower impressions followed by wax bite registrations (maximum intercuspation and protrusive) were made for each patient as previously described in Group I. The upper cast was scanned first using dental scanner (Freedom HD scanner, Germany) followed by scanning both upper and lower casts occluding together by the aid of bite registration. The ARS was designed on upper cast using a software program (Exocad matera 2.3 CAD) that utilized both bite splint module and virtual articulator to provide proper dynamic occlusion. Figure (3a). The major maxillary undercuts on the cast were blocked automatically figure (3b). The blocked model was edited to create retention areas and the design of the splint (the same design of the conventional ARS) was drawn virtually. Dynamic occlusion was set using customizable parameters figure (3c). Occlusal interferences were removed by clicking on "remove occlusion interference" icon. Afterwards, the output file was printed into high cross linked clear hard acrylic resin using rapid prototyping machine (Formlab's 3D printer, United States) figure (3d). The splint was delivered to the patient and checked as previously mentioned. The patient was instructed to wear the splint for 8 hours per day for 6 months. The delivery of CAD/CAM splint was much easier than the conventional type, as it required very little occlusal adjustments (except in two cases who needed few occlusal adjustments).

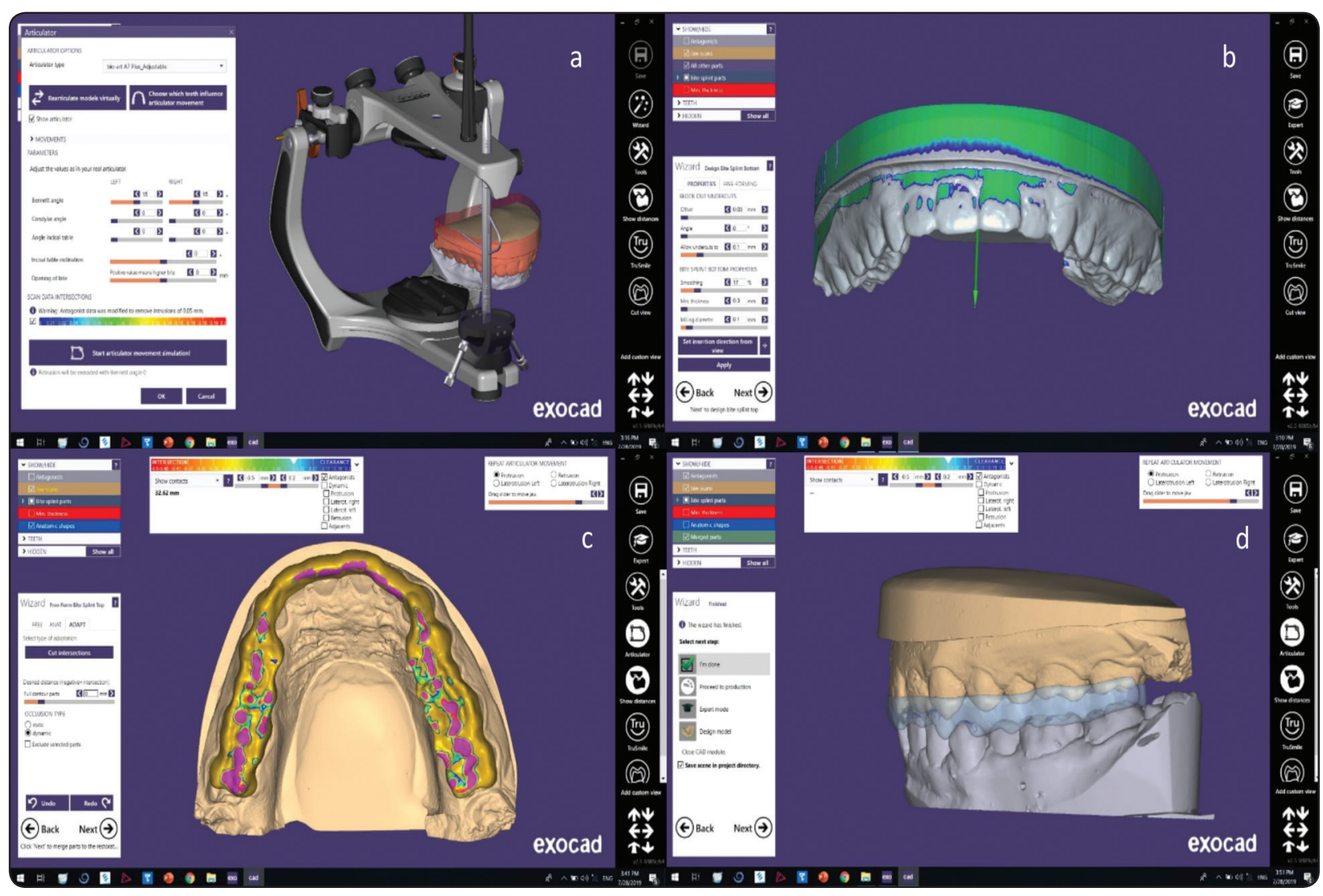

Fig. (3) a) The virtual articulator, b) automatic blocking out of maxillary undercuts, c) the Patient's dynamic occlusion, d) CAD/ CAM splint virtual design sent to the $3 \mathrm{~d}$ printing machine 


\section{Methods of evaluation}

a) TMD Questionnaire (subjective evaluation): The TMD questionnaire was given to the patients to be answered before treatment (pretreatment) and was scheduled again after one month, 3- and 6-months post-insertion of the ARS in both groups.

b) The disc-condyle angle and disc/condyle positions (spatial changes) using MRI: These measurements were made for both normal (control) and DDwR joints within each group. These MRI measurements were obtained and recorded for all joints at three different phases. The first measurements were obtained during diagnosis (pre-treatment) while the patient was closing in maximum intercuspation. The second measurements were obtained immediately after insertion of ARS while each patient was wearing his/her splint (i.e. subjects were directed to open their mouths fully beyond the clicking point and gently bite in protrusive therapeutic position guided by the splint). The third measurements were obtained after 6 months post-insertion (the end of follow-up period) while the patient was closing in maximal intercuspation immediately after splint removal from the patient's mouth (same as the diagnosis position). The MRI measurement results were classified into measurements of the normal (control) joints of both groups together and compared to the DDwR joints in each group (I and II) separately at the three MRI phases (at diagnosis (pre-treatment), immediately postinsertion of the splint and after 6 months post -insertion). Moreover, DDwR joints MRI measurement results in group I (conventional) were compared to those of group II (CAD/CAM) at the same MRI phases.

Results were calculated, tabulated and statistically analyzed using the Minitab 17.0. To assess the VAS scores of the TMD questionnaire in both Groups (I \& II). Mann-Whitney test was used to compare VAS scores of TMD questionnaire between Group I and II in each item in different visits. To compare the MRI measurements (disc-condyle angles, disc and condyle positions) between groups of equal size T-test was used, while when the groups had unequal size Tukey-Kramer test was used. On comparing the MRI measurements within the same group at different MRI phases (at diagnosis (pretreatment), immediately post-insertion of the splint and after 6 months post -insertion) paired t-test was used. Any test value of $\mathrm{p}<0.05$ was considered statistically significant.

\section{RESULTS}

Twenty patients were enrolled in this study; 15 females and 5 males aged 20 to 40 (29.8 26.8 years, mean $\pm \mathrm{SD}$ ). No patient dropped out of the study.

\section{TMD Questionnaire (subjective evaluation)}

There was no significant difference between the scores of VAS in group I as compared to group II $(\mathrm{P}>0.05)$ in all items of TMD questionnaire at all visits; from pretreatment visits till the end of follow-up period (6 months). Obviously from the descriptive statistics in table (2), there was a significant difference (decrease) in the scores of the questionnaire in each item separately within each group between different visits; the scores decreased significantly with time. This indicates decline in severity of subjective symptoms, improvement in quality of patient life, hence ARS in both groups succeeded to decrease the intensity of TMD symptoms and complaints.

\section{The disc-condyle angle and disc/condyle po- sitions (spatial changes) using MRI}

According to the normal range of disc-condyle angle $(-15$ to +15$)$, MRI scan revealed 8 normal joints (out of 20) belonging to ten patients in group I. While MRI scans revealed 6 normal joints (out of 20) in group II. Accordingly, the total number of normal (control) joints in both groups was 14 joints, while the number of DDwR joints in group I and II was 12 was 14 respectively. According to MRI scans results $10(83.33 \%)$ and $12(85.7 \%)$ DDwR joints had their disc successfully recaptured at the end of splint treatment in group I and II respectively. 
The disc-condyle angle in the DDwR joints- that did not have successful disc recapture- approached so closely the normal range $(-15$ to +15$)$.

a) The disc-condyle angle: The mean of pretreatment disc-condyle angles in DDwR joints in group I and II were $52.31^{\circ} \pm 4.59$ and $51.98^{\circ}$ \pm 4.92 respectively figure (1a). These angles were significantly $(\mathrm{p}<0.05)$ greater than the pre-treatment disc-condyle angles of normal joints whose mean was $2.76^{\circ} \pm 4$. figure (1). The therapeutic position (immediate post-insertion) with ARS mean of disc-condyle angles in DDwR joints in group I and group II were $-18.01^{\circ} \pm 3.37$ and $-17.79^{\circ} \pm 3.82$ respectively. At this phase, there was no statistically significant difference between the disc-condyle angle of normal joints and DDwR joints in Group I and II compared separately. Likewise, there was no statistically significant difference between disc-condyle angle in group I and II when compared to each other. After 6 months(post-insertion) the mean disc-condyle angle of DDwR joints in both Group I and II was $5.08^{\circ} \pm 2.01$ and $4.91^{\circ} \pm 2.18$

TABLE (2) TMD Questionnaire VAS scores at different visits in Group I Group II (Mean \pm standard deviation)

\begin{tabular}{|c|c|c|c|c|}
\hline $\begin{array}{l}\text { Items of TMD } \\
\text { questionnaire }\end{array}$ & Evaluation visits & $\begin{array}{c}\text { Group I } \\
\text { (Conventional) } \\
\text { mean } \pm \text { SD } \\
n=20\end{array}$ & $\begin{array}{c}\text { Group II } \\
(\mathrm{CAD} / \mathrm{CAM}) \\
\text { mean } \pm \mathrm{SD} \\
\mathrm{n}=20\end{array}$ & $\begin{array}{c}\text { P-Value } \\
\text { between } \\
\text { Group I and II }\end{array}$ \\
\hline \multirow{4}{*}{$\begin{array}{l}\text { Pain in the joint } \\
\text { around ears }\end{array}$} & Pre-treatment & $79.10 \pm 2.38$ & $79.99 \pm 2.01$ & 0.461 \\
\hline & One Month post-insertion & $64.82 \pm 1.73$ & $65.88 \pm 1.28$ & 0.093 \\
\hline & 3 Months post-insertion & $43.07 \pm 4.98$ & $47.34 \pm 3.01$ & 0.377 \\
\hline & 6 Months post-insertion & $22.13 \pm 2.61$ & $22.42 \pm 2.44$ & 0.212 \\
\hline \multirow{4}{*}{$\begin{array}{l}\text { Pain around the } \\
\text { temple, cheeks, or } \\
\text { jaw }\end{array}$} & Pre-treatment & $81.24 \pm 1.55$ & $80.95 \pm 1.51$ & 0.434 \\
\hline & One Month post-insertion & $65.10 \pm 2.51$ & $63.43 \pm 2.53$ & 0.357 \\
\hline & 3 Months post-insertion & $40.47 \pm 4.93$ & $41.86 \pm 4.40$ & 0.348 \\
\hline & 6 Months post-insertion & $20.83 \pm 2.41$ & $21.85 \pm 2.35$ & 0.480 \\
\hline \multirow{4}{*}{$\begin{array}{l}\text { Sounds during } \\
\text { closing or opening } \\
\text { the jaw; i.e. Crepitus, } \\
\text { Clicking or popping }\end{array}$} & Pre-treatment & $83.50 \pm 1.27$ & $84.23 \pm 1.82$ & 0.496 \\
\hline & One Month post-insertion & $57.42 \pm 1.85$ & $58.62 \pm 1.47$ & 0.325 \\
\hline & 3 Months post-insertion & $33.49 \pm 2.58$ & $32.49 \pm 2.10$ & 0.264 \\
\hline & 6 Months post-insertion & $13.32 \pm 2.99$ & $15.11 \pm 3.36$ & 0.431 \\
\hline \multirow{4}{*}{$\begin{array}{l}\text { Oral parafunctional } \\
\text { habits; i.e. Clenching } \\
\text { or Bruxism }\end{array}$} & Pre-treatment & $75.86 \pm 2.85$ & $75.89 \pm 3.02$ & 0.163 \\
\hline & One Month post-insertion & $41.74 \pm 2.33$ & $42.80 \pm 2.97$ & 0.362 \\
\hline & 3 Months post-insertion & $24.83 \pm 4.33$ & $27.29 \pm 5.80$ & 0.472 \\
\hline & 6 Months post-insertion & $12.07 \pm 1.78$ & $14.33 \pm 3.02$ & 0.206 \\
\hline \multirow{4}{*}{$\begin{array}{l}\text { Difficulties during } \\
\text { opening the mouth }\end{array}$} & Pre-treatment & $51.30 \pm 1.42$ & $52.82 \pm 1.45$ & 0.091 \\
\hline & One Month post-insertion & $36.01 \pm 2.91$ & $37.11 \pm 3.38$ & 0.432 \\
\hline & 3 Months post-insertion & $24.43 \pm 3.07$ & $25.02 \pm 3.14$ & 0.325 \\
\hline & 6 Months post-insertion & $11.48 \pm 2.20$ & $12.48 \pm 2.57$ & 0.237 \\
\hline
\end{tabular}

*P-values $<0.05$ are considered significant. 
TABLE (3) The disc-condyle angle in normal, Group I DDwR and Group II DDwR joints at the three MRI phases (mean \pm standard deviation)

\begin{tabular}{|c|c|c|c|c|c|c|}
\hline & \multirow{2}{*}{$\begin{array}{c}\text { Normal joints } \\
\text { (Control) n=14 } \\
\begin{array}{c}\text { Mean } \pm \text { SD } \\
(\mathrm{mm})\end{array}\end{array}$} & \multicolumn{2}{|c|}{$\begin{array}{c}\text { Group I } \\
\text { DDwR joints (n=12) }\end{array}$} & \multicolumn{2}{|c|}{$\begin{array}{c}\text { Group II } \\
\text { DDwR joints (n=14) }\end{array}$} & \multirow[b]{2}{*}{$\begin{array}{c}\text { p-value } \\
\text { (GrI-Gr II) }\end{array}$} \\
\hline & & $\begin{array}{c}\text { Mean } \pm \text { SD } \\
\quad(\mathrm{mm})\end{array}$ & $\begin{array}{c}\text { p-value } \\
\text { (Normal- } \\
\text { Gr I) }\end{array}$ & $\begin{array}{c}\text { Mean } \pm \text { SD } \\
\quad(\mathbf{m m})\end{array}$ & $\begin{array}{c}\text { p-value } \\
\text { (Normal-Gr } \\
\text { II) }\end{array}$ & \\
\hline Pre-treatment & $2.76^{\circ} \pm 4.21$ & $52.31^{\circ} \pm 4.59$ & 0.0001 & $51.98^{\circ} \pm 4.92$ & 0.0001 & 0.351 \\
\hline $\begin{array}{l}\text { Immediately post- } \\
\text { Insertion }\end{array}$ & $-16.00^{\circ} \pm 4.40$ & $-18.01^{\circ} \pm 3.37$ & 0.351 & $\begin{array}{c}-17.79^{\circ} \pm \\
3.82\end{array}$ & 0.425 & 0.199 \\
\hline $\begin{array}{l}6 \text { Months Post } \\
\text {-Insertion }\end{array}$ & $3.69^{\circ} \pm 2.39$ & $5.08^{\circ} \pm 2.01$ & 0.276 & $4.91^{\circ} \pm 2.18$ & 0.186 & 0.436 \\
\hline
\end{tabular}

$* P$-values $<0.05$ are considered significant

respectively. Hence, the latter disc-condyle angles returned back into the normal range (-15 to +15$)$. There was no statistically significant difference $(\mathrm{p}>0.05)$ in the disc-condyle angle in normal joints when compared with that of DDwR joints in Group I and II separately. Additionally, there was no statistically significant difference $(\mathrm{p}>0.05)$ between the disc-condyle angles in DDwR joints (DDWR) of Group I and Group II at pre-treatment visit, immediately postinsertion and 6 months post-insertion. Table (3)

b) The condyle position: The H-point (center of condylar head) was traced along the X- and Yaxes (coordinates) at the three MRI phases. The change in the condyle position numerically was obtained by the difference between the values of $\mathrm{H}$-point at pre-treatment position and its value at the immediate post-insertion position figure (1b). Results indicated that condylar head of normal joints moved significantly $(\mathrm{p}<0.05)$ in the immediate post-insertion position not only forward (along $\mathrm{X}$ - axis) with an average of $2.86 \mathrm{~mm}$ but also downward (along Y-axis) with an average of $2.01 \mathrm{~mm}$. Additionally, the condylar head of DDWR joints in both group I and group II moved significantly $(\mathrm{p}<0.05)$ forward (along $\mathrm{X}$ - axis) with an average of 1.76 and $1.79 \mathrm{~mm}$ respectively in the immediate post-insertion position as compared to the pretreatment position. Likewise, the condylar head of DDWR joints in both group I and group II moved significantly $(\mathrm{p}<0.05)$ downward (along Y-axis) with an average of 2.79 and $2.78 \mathrm{~mm}$ respectively in the immediate post-insertion position as compared to the pre-treatment position. The change in the condyle position numerically was also obtained by difference between the values of $\mathrm{H}$-point at pre-treatment position and its value at the 6 months postinsertion position. The condylar heads after wearing ARS for 6 months made a significant $(\mathrm{p}<0.05)$ anterior translation (along the $\mathrm{X}$-axes) with an average of $0.85 \mathrm{~mm}$ and $0.77 \mathrm{~mm}$ in DDwR joints of group I and group II respectively as compared to their pre-treatment positions. Likewise, they made a significant $(\mathrm{p}<0.05)$ downward translation (along the Y-axes) with an average of $1.54 \mathrm{~mm}$ and $1.61 \mathrm{~mm}$ in joints of group I and group II respectively as also compared to their pre-treatment positions. However, there were no significant differences in condylar head positions of group I and group II at all MRI phases Table (4). 
TABLE (4) The condyle position along the X-and Y-axes in normal, Group I DDwR and Group II DDwR joints at the three MRI phases (mean \pm standard deviation)

\begin{tabular}{|c|c|c|c|c|c|c|c|}
\hline & & \multirow{2}{*}{$\begin{array}{c}\begin{array}{c}\text { Normal joints } \\
\text { (Control) } \\
n=14\end{array} \\
\begin{array}{c}\text { Mean } \pm \text { SD } \\
(\mathrm{mm})\end{array}\end{array}$} & \multicolumn{2}{|c|}{$\begin{array}{c}\text { Group I } \\
\text { DDwR joints } \\
\text { n=12 }\end{array}$} & \multicolumn{2}{|c|}{$\begin{array}{c}\text { Group II } \\
\text { DDwR joints } \\
\text { n=14 }\end{array}$} & \multirow[t]{2}{*}{$\begin{array}{c}\text { p-value } \\
\text { (Gr I-Gr II) }\end{array}$} \\
\hline & & & $\begin{array}{c}\text { Mean } \pm \text { SD } \\
(\mathrm{mm})\end{array}$ & $\begin{array}{c}\text { p-value } \\
\text { (Normal-Gr I) }\end{array}$ & $\begin{array}{c}\text { Mean } \pm \text { SD } \\
(\mathrm{mm})\end{array}$ & $\begin{array}{c}\text { p-value } \\
\text { (Normal-Gr II) }\end{array}$ & \\
\hline \multirow{2}{*}{ Pre- treatment } & $\mathrm{X}$-axis & $0.19 \pm 1.61$ & $1.01 \pm 0.47$ & 0.0001 & $1.03 \pm 0.45$ & 0.0001 & 0.259 \\
\hline & Y-axis & $-6.79 \pm 1.78$ & $-6.50 \pm 1.24$ & 0.0001 & $-6.40 \pm 1.35$ & 0.0001 & 0.365 \\
\hline \multirow{2}{*}{$\begin{array}{c}\text { Immediately } \\
\text { After Insertion }\end{array}$} & $\mathrm{X}$-axis & $-3.05 \pm 1.85$ & $-2.89 \pm 0.28$ & 0.475 & $-2.79 \pm 0.39$ & 0.199 & 0.453 \\
\hline & Y-axis & $-8.80 \pm 1.12$ & $-9.29 \pm 2.56$ & 0.254 & $-9.18 \pm 2.97$ & 0.231 & 0.432 \\
\hline \multirow{2}{*}{$\begin{array}{c}6 \text { Months Post } \\
\text { Insertion }\end{array}$} & $\mathrm{X}$-axis & $-0.12 \pm 1.58$ & $-0.16 \pm 0.38$ & 0.387 & $-0.26 \pm 0.25$ & 0.126 & 0.238 \\
\hline & Y-axis & $-7.76 \pm 1.24$ & $-8.04 \pm 2.38$ & 0.199 & $-8.01 \pm 2.43$ & 0.315 & 0.427 \\
\hline
\end{tabular}

*P-values $<0.05$ are considered significant

c) The Disc position: The P-point (the mid-point of the posterior margin of the disc) denoted the disc positions in the $\mathrm{X}$ - and $\mathrm{Y}$ - axes (coordinates). This point was traced in images at the three MRI phases. In the joints with DDWR, the immediate splint insertion caused discs to move significantly $(\mathrm{p}<0.05)$ both backward ( along the $\mathrm{X}$-axis) with an average of 2.78 and 2.85 $\mathrm{mm}$ in group I and group II respectively, and upward (along the Y-axis) with an average of 0.60 and $0.62 \mathrm{~mm}$. The change in disc position was obtained by the difference between the disc position(P-Point) in the pre-treatment images and the disc position at the immediate post-insertion images in both groups. After 6 months (post-insertion), on comparing with the pre-treatment position, the discs at maximum intercuspation moved significantly $(\mathrm{p}<0.05)$ both backward (along $\mathrm{X}$-axis) with an average of $2.35 \mathrm{~mm}$ and $2.33 \mathrm{~mm}$ in the DDwR joints of group I and group II respectively, and upward (along Y-axis) with an average of 1.12 and 1.14 $\mathrm{mm}$ in the DDwR joints of group I and group II respectively Table (5). Discs in the normal joints did not show significant ( $p>0.05$ ) change in position between the three MRI phases in both $\mathrm{x}$ and Y-axes , as the P-point was normally located about 0 to $1 \mathrm{~mm}$ along $\mathrm{X}$-axis behind the vertex of glenoid fossa (F-point).There was a statistically significant difference $(\mathrm{p}<0.05)$ between the disc positions in normal joints and DDwR joints of group I and II at the pretreatment phase compared separately. On the contrary, there was a no statistically significant difference between the disc positions in normal joints and DDwR joints of group I and II after 6 months post-insertion phase compared separately. The discs positions in group I DDwR joints expressed no significant difference when compared to DDwR joints group II at the three MRI phases. 
TABLE (5) The disc position along the X-and Y-axes in normal, Group I and Group II at the three MRI phases (mean \pm standard deviation)

\begin{tabular}{|c|c|c|c|c|c|c|c|}
\hline & & \multirow{2}{*}{$\begin{array}{c}\text { Normal joints } \\
\begin{array}{c}\text { (Control) } n=14 \\
\text { Mean } \pm \text { SD } \\
(\mathrm{mm})\end{array}\end{array}$} & \multicolumn{2}{|c|}{$\begin{array}{c}\text { Group I } \\
\text { DDwR joints } \\
\text { n=12 }\end{array}$} & \multicolumn{2}{|c|}{$\begin{array}{c}\text { Group II } \\
\text { DDwR joints } \\
\mathbf{n}=14\end{array}$} & \multirow{2}{*}{$\begin{array}{c}\text { p-value } \\
\text { (Gr I -Gr II) }\end{array}$} \\
\hline & & & $\begin{array}{c}\text { Mean } \pm \mathrm{SD} \\
(\mathrm{mm})\end{array}$ & $\begin{array}{c}\text { p-value } \\
\text { (Normal-Gr I) }\end{array}$ & $\begin{array}{c}\text { Mean } \pm \text { SD } \\
(\mathrm{mm})\end{array}$ & $\begin{array}{c}\text { p-value } \\
\text { (Normal-Gr II) }\end{array}$ & \\
\hline \multirow{2}{*}{ Pre-treatment } & $\mathrm{X}$-axis & $0.60 \pm 0.91$ & $-3.10 \pm 1.32$ & 0.0001 & $-3.13 \pm 1.28$ & 0.0001 & 0.342 \\
\hline & Y-axis & $-1.60 \pm 0.52$ & $-3.79 \pm 1.39$ & 0.0001 & $-3.84 \pm 1.34$ & 0.0001 & 0.467 \\
\hline \multirow{2}{*}{$\begin{array}{l}\text { Immediately } \\
\text { After Insertion }\end{array}$} & $\mathrm{X}$-axis & $0.71 \pm 1.27$ & $0.32 \pm 1.06$ & 0.189 & $0.28 \pm 1.10$ & 0.234 & 0.481 \\
\hline & Y-axis & $-2.70 \pm 0.72$ & $-3.19 \pm 1.32$ & 0.158 & $-3.22 \pm 1.29$ & 0.215 & 0.415 \\
\hline \multirow{2}{*}{$\begin{array}{l}6 \text { Months Post } \\
\text { Insertion }\end{array}$} & $\mathrm{X}$-axis & $0.81 \pm 1.39$ & $-0.75 \pm 1.88$ & 0.354 & $-0.80 \pm 1.82$ & 0.478 & 0.176 \\
\hline & Y-axis & $-2.02 \pm 1.15$ & $-2.67 \pm 0.91$ & 0.243 & $-2.70 \pm 0.88$ & 0.126 & 0.167 \\
\hline
\end{tabular}

*P-values $<0.05$ are considered significant

\section{DISCUSSION}

Conservative treatment options in the management of TMDs are massage, exercises, counselling, manual therapy occlusal splint therapy and medications. These options are considered as an initial choice for TMD therapy due to their low risk of side effects. The ideal treatment method for disc displacement is still controversial. One of the most commonly used occlusal splint is the anterior repositioning splint (ARS). The success of ARS to re-establish correct disc-condyle relationships in joints with DDwR and its superiority to other splints was reported in several studies..$^{710,25}$ ARS use in these joints facilitated regenerative remodeling of condyles. ${ }^{13}$ However, most of these researches were based on improvement of subjective symptoms especially incidence of pain. MRI metrical measurements and analysis provide a more reliable method enabling both qualitative and quantitative evaluation of structures within the joint, including the TMJ disc and condyle positions. MRI is a painless non-invasive and safe method, as it does not use ionizing radiation. ${ }^{14}$ When ARS splint is inserted, the jaws become adapted to a new freeway space with an increased vertical dimension that occurs as a result of even thickness of splint occlusal surface $(4 \mathrm{~mm})$. This high vertical dimension provides muscle relaxation during resting position and hence relieving pain as well as allowing efficient muscular contraction during function ${ }^{6}$. Metrical analysis of MRI scan provides an accurate view of the mechanism of the splint revealed in maximum intercuspation position. In the current study, the discs in DDwR joints were displaced anteriorly and inferiorly, while the condyle was positioned backward and upward, in relation to normal joints. In ARS treatment positions caused forward and downward movement of the condyle, additionally ARS produced a significant posterior movement of the disc and similar significant anterior movement of the condyle resulting in recapture of the disc and then gradual withdrawal of the disc-condyle complex into the glenoid fossa; this mechanism of ARS explains its superiority in disc recapturing over any other splint and clarifies the importance of MRI scans in any study concerned with physiological effect of occlusal splints on TMJ. These results 
were in accordance with several clinical trials investigating the changes in the disc/condyle position with ARS ${ }^{10,14,31}$. The current study relied on MRI scans as well as TMD questionnaire (subjective symptoms) to make an accurate comparative evaluation of CAD $\backslash C A M$ versus conventional ARS. The clinical trials that used the MRI results to confirm the clinical evaluation of splint therapy are scarce in literature. Moreover, the current study may be one of extremely rare studies comparing the efficacy of CAD/CAM versus conventional ARS in treatment of disc displacement with reduction cases of TMD utilizing MRI to confirm the results of TMD questionnaire.

The success rate in the current study was $83.33 \%$ and $85.71 \%$ in Group I and Group II respectively. This can be attributed to the meticulous follow to the construction procedures of ARS (especially mounting on articulators and precise occlusal adjustments). The same success rate was observed with a similar clinical study investigating the effect of differently designed occlusal splints on disc recapturing in TMD patients. ${ }^{31}$ On the other hand, this success rate was different from another study ${ }^{10}$ which reported that $40.6 \%$ of the DDwR joints were successfully repositioned. This can be attributed to fact that patients in the latter study wore a conventionally constructed ARS continuously for 3 months then during the next 3 months they wore it during sleep only. This was different from the current study where the patients wore the splint for 8 hours daily for 6 months. Additionally, the strict follow to construction procedures of ARS in both group and the use of CAD/CAM technique in the current study may have led to the higher success rate.

The significant decrease in the TMD questionnaire items scores within each group indicated significant improvement of all subjective annoying symptoms along follow-up periods compared to pre-treatment (baseline) scores. The MRI results augmented the results of TMD questionnaire indicating that proper disc-condyle relation attained by ARS can eliminate joint sounds and alleviate pain. This superior efficacy of ARS in relieving TMD symptoms was also confirmed in other studies ${ }^{7,9,30}$ and explained by MRI metric analysis done in rare studies that measured the changes on disc and condyle spatial position following treatment with $\mathrm{ARS}^{8,10,14}$.

There was no significant difference between the TMD questionnaire item scores and MRI metric analysis of CADICAM versus conventional ARS at all follow-up visits. This indicates equivalent success of both types of splints and comparable effectiveness in the management of DDwR cases of TMD. This outcome was obtained probably as a result of following strict standard prosthetic procedures especially during jaw relation records that were transferred to the articulator to mimic the virtual articulator of CADICAM machine, moreover all precautions were taken during processing of conventional splints to avoid any mistake that may affect either stability or occlusal scheme of splint . However, in the current clinical trial CAD CAM technique saved chair-side time during prosthetic construction, and occlusal adjustments. The main benefit of CADICAM technology was the elimination of human errors inherent in the processing of polymethyl-methacrylate including over contour and polymerization shrinkage that would have resulted in poor fit and irregular occlusal scheme in conventionally -fabricated splints. ${ }^{18-21}$

In Intra-group results of MRI measurements in the current study revealed that immediate insertion of ARS (either conventional or CADICAM ) restored the disc-condyle angle of DDwR joints in Group I and II at the protrusive position as compared to the angles of that of normal joints, as the condyles moved significantly forward and downward, while the discs moved significantly backward with splint. This are the same results obtained from another MRI metrical analysis study ${ }^{14}$. At 6 months after treatment, the majority of discs in DDwR joints in Group I and II were recaptured indicating successful treatment. These results are consistent with the results reported by another clinical study evaluating the physiological effect of ARS on disc-condyle position using $\mathrm{MRI}^{31}$. 


\section{CONCLUSION}

Within the limitations of this study, there was no significant difference between the effect of CAD/ $\mathrm{CAM}$ versus conventional ARS on the management of DDwR. Hence, the CAD/CAM ARS can provide a good substitute to the conventional ARS in treatment of DDwR eliminating human errors and saving time. Moreover, the superior effect of ARS in the management of DDwR could be related mainly to the design of the splint rather than the method involved in the construction of the splint.

\section{REFERENCES}

1. Ahmad M, SchiDman EL. Temporomandibular joint disorders and orofacial pain. Dent Clinic North America. 2016; 60:105-124.

2. SchiDman E, Ohrbach R, Truelove E, et al. Diagnostic criteria for temporomandibular disorders (DC/TMD) for clinical and research applications: recommendations of the International RDC/TMD Consortium Network and Orofacial Pain Special Interest Group. J Oral Fac Pain Headache, 2014; 28: 6-27.

3. Al-Khotani A, Naimi-Akbar A, Albadawi E, Ernberg M, Hedenberg-Magnusson B, Christidis N. Prevalence of diagnosed temporomandibular disorders among Saudi Arabia children and adolescents. J of Headache and Pain, 2016; 1: 1-11.

4. Kumazaki Y, Kawakami S, Hirata A, Oki K, Minagi S. Ipsilateral molar clenching induces less pain and discomfort than contralateral molar clenching in patients with unilateral anterior disc displacement of the temporomandibular joint. $\mathrm{J}$ of Oral and Facial Pain and Headache, 2016; 241:248 -30

5. Karacayli U, Mumcu G, Cimilli H, Sisman N, Sur H, and Gunaydin Y. The effects of chronic pain on oral health related quality of life in patients with anterior disc displacement with reduction. Community Dental Health, 2011; 28: 211-215.

6. Amin A , Meshramkar R and Lekha K. Comparative evaluation of clinical performance of different kind of occlusal splint in management of myofascial pain. The J of Indian Prosthodontic Society. 2016; 16: 176-181.

7. Pihut M, Gorecka, M, Ceranowicz, P and Wieckiewicz M. The Efficiency of Anterior Repositioning Splints in the Management of Pain Related to Temporomandibular Joint Disc Displacement with Reduction. Pain Res and Management, 2018;10:1155-1161.

8. Eberhard D, Bantleon H. P and Steger W. The efficacy of anterior repositioning splint therapy studied by magnetic resonance imaging. European Journal of Orthodontics, 2002; 24:343-352.

9. Tecco S, Festa F, Salini V, Epifania E and D'Attilio M. Treatment of joint pain and joint noises associated with a recent TMJ internal derangement: A comparison of an anterior repositioning splint, a full-arch maxillary stabilization splint, and an untreated control group. Cranio, 2004;22:209-219.

10. Chen, H. M, Liu M. Q, Yap A. U and Fu KY. Physiological effects of anterior repositioning splint on temporomandibular joint disc displacement: a quantitative analysis. J Oral Rehab. 2017; 44: 664-672.

11. Okeson JP, Management of temporomandibular disorders and occlusion. 7th ed. Saint Louis: Mosby; 2012.

12. Boero RP. The physiology of splint therapy: a literature review.Angle Orthod, 1989; 59: 165-80.

13. Liu MQ, Chen HM, Yap AU, Fu KY. Condylar remodeling accompanying splint therapy: a cone-beam computerized tomography study of patients with temporomandibular joint disk displacement. Oral Surg Oral Med Oral Pathol Oral Radiol. 2012; 114: 259-265.

14. Liu M Q , Lei J , Han J H , Yap A U and Fu KY. Metrical analysis of disc-condyle relation with different splint treatment positions in patients with TMJ disc displacement. J of Appl Oral Sci, 2017; 25: 48 -489.

15. Conti PC, Corrêa AS, Lauris J, Stuginski-Barbosa J. Management of painful temporomandibular joint clicking with different intraoral devices and counseling: A controlled study. J Appl Oral Sci 2015; 23:529-535.

16. Abduo J, Lyons K, Bennamoun M. Trends in computeraided manufacturing in prosthodontics: a review of the available streams. Int J Dent. 2014; 8: 1-15.

17. Stansbury J W and Idacavage M J, 3D printing with polymers: challenges among expanding options and opportunities. Dent. Mater, 2016; 54:64-32.

18. Dedem P, Turp J C. Digital Michigan splint - from intraoral scanning to plasterless manufacturing. Int J Comput Dent, 2016;19: 63-76. 
19. Dunn DB, Lewis MB, CAD/CAM occlusal splints: A new paradigm. Aust Dent Pract, 2011;22:131-9.

20. Hogan J, DentaBite: A precision engineering solution to a traditional problem. Aust Dent Pract 2011; 8:164-8.

21. Pho Duc J M, Hüning S V and Grossi M L. Parallel Randomized Controlled Clinical Trial in Patients with Temporomandibular Disorders Treated with a CAD/CAM Versus a Conventional Stabilization Splint. Int J Prosthodont,2016;29:340-350.

22. Algabri RS, Alqutaibi AY, Abo-Alrejal H, Al Adashi O, Abdulrahman F, Elkhadem A, Keshk A, Maher E, Kaddah AF. Effect of computer-aided design/computer-assisted manufacture versus conventional occlusal splints on the management of temporomandibular disorders: A systematic review and meta-analysis. Int Dent Med J Adv Res 2017; 3:1-9.

23. Fricton JR, Ouyang W, Nixdorf DR, Schiffman EL, Velly AM, Look JO. Critical appraisal of methods used in randomized controlled trials of treatments for temporomandibular disorders. J Orofac Pain. 2010; 24:139-151.

24. Kurita H, Ohtsuka A, Kurashina K and Kopp S A, study of factors for successful splint capture of anteriorly displaced temporomandibular joint disc with disc repositioning appliance. J Oral Rehab. 2001; 651:657- 28

25. Ma Z, Xie Q, Yang C, Zhang S, Shen Y, Ahmed A. Can anterior repositioning splint effectively treat temporomandibular joint disc displacement? Scientific Reports. 2019; 9:534-50.
26. GonÇalves DA, Dal Fabbro AL, Campos JA, Bigal ME Speciali JG. Symptoms of temporomandibular disorders in the population: an epidemiological study. J Orofac Pain. 2010; 24:270-8.

27. Svensson P, Lars A-N, Nielsen, Larsen H, Jonna K. Effect of Chronic and Experimental Jaw Muscle Pain on PainPressure Thresholds and Stimulus-Response Curves. J of Orofacial Pain. 1995; 9: 347-356.

28. Drace JE, Enzmann DR. Defining the normal temporomandibular joint: closed-, partially open-, and open-mouth MR imaging of asymptomatic subjects. Radiology. 1990; 177:67-71.

29. Ahmad M, Hollender L, Anderson Q, Kartha K, Ohrbach $\mathrm{R}$, Truelove EL, et al. Research diagnostic criteria for temporomandibular disorders (RDC/TMD): development of image analysis criteria and examiner reliability for image analysis. Oral Surg Oral Med Oral Pathol Oral Radiol Endod. 2009; 107: 844-60.

30. Zhang C, Wu JY, Deng DL, He BY, Tao Y, Niu YM, Deng MH. Efficacy of splint therapy for the management of temporomandibular disorders: a meta-analysis. Oncotarget. 2016;7(51): 84043-53.

31. Yang Ch, Shen P. Assessment of occlusal appliance in repositioning of the temporomandibular joint anterior disc displacement with reduction: a 3 to 36 months follow-up. Almanac of Clinical Medicine. 2017;45(6):478-85. 